\title{
Editorial
}

\section{The archaeology of apoptosis}

\author{
RA Knight ${ }^{*, 1}$ \\ 1 Department of Cystic Fibrosis, National Heart and Lung Institute, Imperial \\ College, Manresa Road, London SW3 6LR \\ * Corresponding author: RA Knight, Department of Cystic Fibrosis, National \\ Heart and Lung Institute, Imperial College, Manresa Road, London SW3 6LR \\ Fax: +44 (0)207 351 8331; E-mail: r.a.knight@ic.ac.uk
}

Cell death with at least some of the morphological and molecular features of apoptosis has been described previously in unicellular organisms such as Trypanosomatids, ${ }^{1,2}$ Tetrahymena ${ }^{3}$ and Dictyostelium. ${ }^{4}$ Two papers in this issue of CDD, ${ }^{5,6}$ studying Leishmania donovani and L. major respectively, extend considerably our understanding of this cell death process in single celled eukaryotes, and reinforce the idea that apoptosis may not simply have evolved with multicellularity. When dealing with cell death in unicellular organisms, however, we need to keep three things in mind. Firstly, although it is tempting, and indeed reassuring, to think that our scientific interests have the respectability of a billionyear history, we need to be aware of the differences, as well as the similarities between cell death in unicellular and multicellular organisms. Secondly, although we are beginning to understand the physiological and pathological significance of apoptosis in the context of multicellularity, we must now try to assess what apoptosis might mean biologically for unicellular organisms. And finally, since we, as investigative multicellular organisms ourselves, cannot merely observe but must also meddle and manipulate, we need to consider the implications of death in unicellular organisms for the hostparasite relationship, and for our ability to influence it therapeutically.

As a student, I was taught that 'life' has no simple definition, but is perhaps best understood as a set of characteristics like respiration, reproduction, growth etc. As in 'life', so in 'death'. We characterise apoptosis in such terms as its morphology, mitochondrial damage, caspase activation, DNA fragmentation and so on. So how well does death in Leishmania measure up to these criteria?

With stationary L. donovani promastigotes, Lee et al. find nuclear condensation with oligonucleosomal DNA fragmentation and TUNEL positivity, together with loss of the mitochondrial membrane potential. In addition, the dying promastigotes express an enzymatic activity which cleaves the caspase substrate, PPL, although this is only inhibited by up to $34 \%$ by conventional caspase inhibitors. In staurosporine-treated $L$. major, the picture is perhaps not quite so clear. Although EM reveals chromatin condensation, and the DNA is fragmented, there does not appear to be classical oligonucleosomal laddering. Staurosporine treatment causes loss of mitochondrial membrane potential, which is associated with cytochrome $c$ release. Again, broad spectrum caspase inhibitors reduce the DNA fragmentation (and the rather untypical PARP and ICAD cleavage that is catalysed), but, as in the $L$. donovani study, do not affect $\Delta \Psi_{\mathrm{m}}$. This suggests that these caspase-like enzymes are activated downstream of mitochondrial injury, just like our dear old caspase- 9 .

Of great practical importance here is the detection of Leishmania cytochrome $c$ using an antibody raised against the corresponding Saccharomyces protein, although antibodies specific for Dictyostelium AIF do not react with Leishmania AIF. This suggests that species cross reactivity may be of some use in dissecting death pathways in unicellular eukaryotes, although more specific reagents are clearly needed. Nowhere is this more important than in the precise identification of the Leishmania caspase-like activity. Is it a true caspase, a meta/paracaspase or something else? One enzyme or many? What is its relationship to the caspases of $C$. elegans, Drosophila and vertebrates? Nevertheless, an impressive list of criteria has already been assembled to allow the provisional award of the title apoptosis to these unicellular death phenomena.

Clearly, any suggestions on the relevance of an inbuilt death mechanism to Leishmania itself can only be tentative. One idea is that, as in multicellular organisms, apoptosis acts to regulate the total population of these unicellular eukaryotes. The finding that stationary Leishmania promastigotes die in vitro may perhaps reflect the in vivo death of excess promastigotes once the limiting nutritional resources of the gut of their sand fly host are approaching exhaustion - a process that may be mediated by signalling between individual promastigotes or follow signals derived from the host. However, it would seem energetically disadvantageous to produce excess progeny and then require them to die by an active process if nutrient supply is the critical factor determining promastigote numbers. A further possibility is that, once a certain proportion of cells have differentiated into infectious metacyclic forms, remaining uninfectious parasites die since they no longer contribute to the perpetuation of the Leishmania life cycle, and indeed may compete with the differentiated Leishmania for available nutrients. This hypothesis would therefore predict that promastigote apoptosis in the sand fly only occurs after some metacyclic forms have appeared. Does the possibility of death/survival signalling between promastigotes or between differentiated and undifferentiated forms imply that we are not dealing here with socially isolated single cells, but rather with a coordinated colony - a precursor of true multicellularity? No Leishmania is an island?

After infection of mammalian hosts, Leishmania amastigotes live in macrophage phagolysosomes. Arnoult et al. discuss the evidence that macrophage ingestion of apoptotic mammalian cells reduces their secretion of proinflammatory cytokines, and offer the interesting specula- 
tion that the ability of intracellular Leishmania to undergo apoptosis may, similarly, reduce the host immune response and favour overall parasite survival. Indeed, phagocytosis of apoptotic cells has also been reported to favour the intracellular growth of Trypanosoma cruzi. ${ }^{7}$ However, data in the Lee et al. paper show that common anti-Leishmanial drugs also induce PPL cleavage activity and DNA fragmentation in $L$. donovani amastigotes in vitro. Do these drugs also induce intracellular apoptosis of some Leishmania, and does this facilitate growth of the survivors? Should we be thinking of developing drugs that cause necrotic death of Leishmania, since ingestion of necrotic material has been shown not to be immunosuppressive?

One major advantage for the evolutionary archaeologist over the diggers and daters of conventional archaeology is the ability to study directly the biology of organisms which have survived for millennia, rather than having to make inferences from artefacts whose survival owes much to chance. We can do better than indulge in inconclusive debates as to whether Stonehenge represents a 5000 yearold version of the Hubble telescope. In Leishmania, we have an existing descendant of an ancient life form which has already evolved a form of apoptotic programme facilitating its adaptation to a digenic life cycle. Thus, Leishmania apoptosis may both maximise the biological fitness of the promastigote colony and reduce the severity of immune assault on mammalian intracellular amastigotes. However, we still need closer and more detailed observation of this death process to allow us to intervene more effectively in clinical parasitic disease.

1. Welburn SC, Dale C, Ellis D, BeecroftR Rand Pearson TW (1996) Cell Death Differ. 3: $229-236$

2. Ameisen JC (1996) Science 272: 1278-1279

3. Davis MC, Ward JG, Herrick G and Allis CD (1992) Dev. Biol. 154: 419-432

4. Cornillon S, Foa C, Davoust J, Buonavista N, Gross JD and Goldstein P (1994) J. Cell. Sci. 107: $2691-2704$

5. Lee N, Bertholet S, Debrabant A, Muller J, Duncan R and Nakhasi HL (2002) Cell Death Differ. 9: 53-64

6. Arnoult D, Akarid K, Grodet A, Petit PX, Estaquier J and Ameisen JC (2002) Cell Death Differ. 9: 65-81

7. Freire-de-Lima CG, Nascimento DO, Soares MBP, Bozza PT, Castro-Faria-Neto HC, de Mello FG, DosReis GA and Lopes MF (2000) Nature 403: 199-203 